\title{
Ischemic enteritis with intestinal stenosis
}

\author{
Yorimitsu Koshikawa ${ }^{1}$, Hiroshi Nakase ${ }^{1}$, Minoru Matsuura ${ }^{1}$, Takuya Yoshino ${ }^{1}$, Yusuke Honzawa ${ }^{1}$, Naoki \\ Minami ${ }^{1}$, Satoshi Yamada ${ }^{1}$, Yumiko Yasuhara ${ }^{2}$, Shigehiko Fujii ${ }^{3}$, Toshihiro Kusaka ${ }^{3}$, Dai Manaka ${ }^{3}$, Hiroyuki \\ Kokuryu $^{3}$ \\ ${ }^{1}$ Department of Gastroenterology and Hepatology, Graduate School of Medicine, Kyoto University, ${ }^{2}$ Department of Pathology, Kyoto Katsura \\ Hospital, ${ }^{3}$ Digestive Disease Center, Kyoto Katsura Hospital, Kyoto, Japan
}

A 75-year-old man was admitted to our hospital with sudden onset of vomiting and abdominal distension. The patient was taking medication for arrhythmia. Computed tomography showed stenosis of the ileum and a small bowel dilatation on the oral side from the region of stenosis. A transnasal ileus tube was placed. Enteroclysis using contrast medium revealed an approximately $6-\mathrm{cm}$ afferent tubular stenosis $10 \mathrm{~cm}$ from the terminal ileum and thumbprinting in the proximal bowel. Transanal double-balloon enteroscopy showed a circumferential shallow ulcer with a smooth margin and edema of the surrounding mucosa. The stenosis was so extensive that we could not perform endoscopic balloon dilation therapy. During hospitalization, the patient's nutritional status deteriorated. In response, we surgically resected the region of stenosis. Histologic examination revealed disappearance of the mucosal layer and transmural ulceration with marked fibrosis, especially in the submucosal layer. Hemosiderin staining revealed sideroferous cells in the submucosal layers. Based on the pathologic findings, the patient was diagnosed with ischemic enteritis. The patient's postoperative course was uneventful. (Intest Res 2016;14:89-95)

Key Words: Ischemia; Intestines; Stenosis; Double-balloon enteroscopy

\section{INTRODUCTION}

Ischemic enteritis (IE) occurs when arterial inflow to the small intestine is reduced. The frequency of IE is very low compared with cases of ischemic colitis (IC) owing to the rich number of arteries supplying the small intestine, and thus there are no established clinical or pathologic diagnostic criteria for IE. In addition, due to the relative difficulty in examining the small intestine, the characteristics of IE remain unclear. Unlike IC, which usually runs a benign course,

Received February 23, 2015. Revised July 7, 2015. Accepted July 13, 2015. Correspondence to Hiroshi Nakase, Department of Gastroenterology and Hepatology, Graduate School of Medicine, Kyoto University, 54 Kawaharacho, Shogoin, Sakyo-ku, Kyoto 606-8507, Japan. Tel: +81-75-751-4319,

Fax:+81-75-751-4303, E-mail: hiropy_n@kuhp.kyoto-u.ac.jp

Financial support: This work was supported by the Japanese Society for the Promotion of Science ("KAKENHI" grant numbers 24590941, 25860532, and 26460967) and in part by Health and Labour Sciences Research Grants for research on intractable diseases from the Ministry of Health, Labour and Welfare of Japan (Investigation and Research for Intractable Inflammatory Bowel Disease). Conflict of interest: None. most cases of IE are accompanied by small intestinal obstruction and require surgical treatment. Herein we report a rare case of IE with intestinal obstruction.

\section{CASE REPORT}

A 75-year-old man was admitted to our hospital with sudden onset of vomiting and abdominal distension. He also complained of mild and intermittent abdominal pain. He reported taking disopyramide and diltiazem for arrhythmia and no history of abdominal surgery. On admission, the patient's blood pressure was 90/60 $\mathrm{mmHg}$, heart rate was 80 beats $/$ min, body temperature was $36.7^{\circ} \mathrm{C}$, and the in-room oxygen saturation was set to $97 \%$. Physical examination revealed mild tenderness in the left side of the abdomen. His abdomen was distended and bowel sounds were hyperactive with metallic sounds. There were no signs of peritoneal irritation. Abdominal radiography showed small bowel dilation (Fig. 1A) and CT showed stenosis of the ileum and a small bowel dilatation on the oral side from the region of

\footnotetext{
(c) Copyright 2016. Korean Association for the Study of Intestinal Diseases. All rights reserved.

This is an Open Access article distributed under the terms of the Creative Commons Attribution Non-Commercial License (http://creativecommons.org/licenses/by-nc/4.0)

which permits unrestricted non-commercial use, distribution, and reproduction in any medium, provided the original work is properly cited.
} 

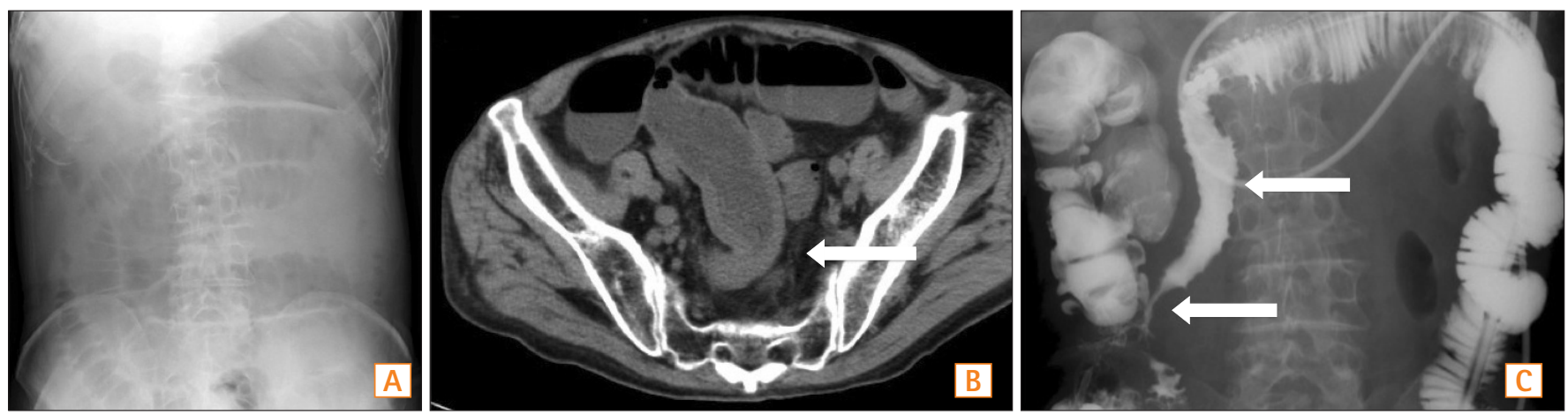

Fig. 1. Radiologic findings on admission and on post-admission day 14. (A) Abdominal radiography on admission showed marked small bowel dilation. (B) Abdominal CT scan showed thickened wall of ileum and stenosis (arrow) and small bowel dilatation. (C) A small intestinal series revealed afferent tubular stenosis of ileum (yellow arrow) and thumbprinting of the oral ileum (arrow).

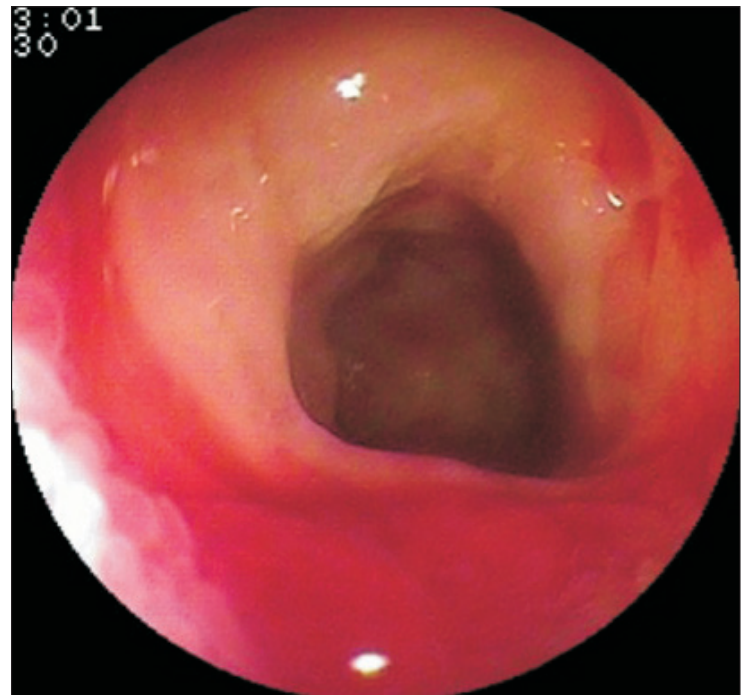

Fig. 2. Endoscopic findings on post-admission day 25. Double balloon enteroscopy (DBE) showed a circumferential ulcer and edematous mucosa. The endoscope could not pass through the stenosis.

stenosis (Fig. 1B). Laboratory findings revealed a slightly elevated CRP level ( $1.9 \mathrm{mg} / \mathrm{dL}$; normal, $0-0.3 \mathrm{mg} / \mathrm{dL}$ ) and hypoalbuminemia ( $2.2 \mathrm{~g} / \mathrm{dL}$; normal, 3.8-5.2 g/dL). A transnasal ileus tube was placed for decompression of the small intestinal obstruction. Enteroclysis using contrast medium through the tube on post-admission day 14 revealed an approximately $6-\mathrm{cm}$ afferent tubular stenosis $10 \mathrm{~cm}$ from the terminal ileum on the oral side and thumbprinting in the proximal bowel (Fig. 1C).

We performed retrograde double-balloon enteroscopy on post-admission day 25 , revealing a circumferential shallow ulcer with a smooth margin and edema of the surrounding mucosa (Fig. 2). The biopsy specimen from the ulcer did not show malignancy. Both PCR for tuberculosis of the specimen and interferon-gamma releasing assays (QuantiFERON ${ }^{\circledR}$-TB Gold In-Tube; Cellestis, Carnegie, VIC, Australia) were negative. We did not perform endoscopic balloon dilatation (EBD) because of the length of the stenosis. During hospitalization, the patient's nutritional status deteriorated. In response, we surgically resected the region of stenosis on post-admission day 30. Histologic examination revealed disappearance of the mucosal layer and transmural ulceration with marked fibrosis, especially in the submucosal layer. Hemosiderin staining revealed sideroferous cells in the submucosal layers. Granuloma was not detected (Fig. 3). Based on findings from the resected specimen, the patient was diagnosed with IE. The patient's postoperative course was uneventful.

\section{DISCUSSION}

IC develops due to hypoperfusion of mesenteric vessels in the absence of major vessel occlusion and usually runs a benign course after acute colonic insult. ${ }^{1}$ IE is termed as the same condition in the small intestine. Most patients with IE, on the contrary, eventually require surgical treatment to alleviate their symptoms. Thus, IE is a rare disease with an unfavorable clinical outcome due to its irreversible pathophysiology. IE is classified into two types: stenotic and transient. ${ }^{2}$ In clinical practice, the transient type is usually misdiagnosed because it resolves in a few days. Therefore, most case reports of IE are classified as the stenotic type. Raf reported that only 11 cases $(0.1 \%)$ of ischemic stenosis of the small intestine were detected among 9,536 patients undergoing surgical resection of the small intestine. ${ }^{3}$ However, it is difficult to estimate the real incidence of patients with either type of IE.

PubMed and Ichushi-Web were used to collect previous 


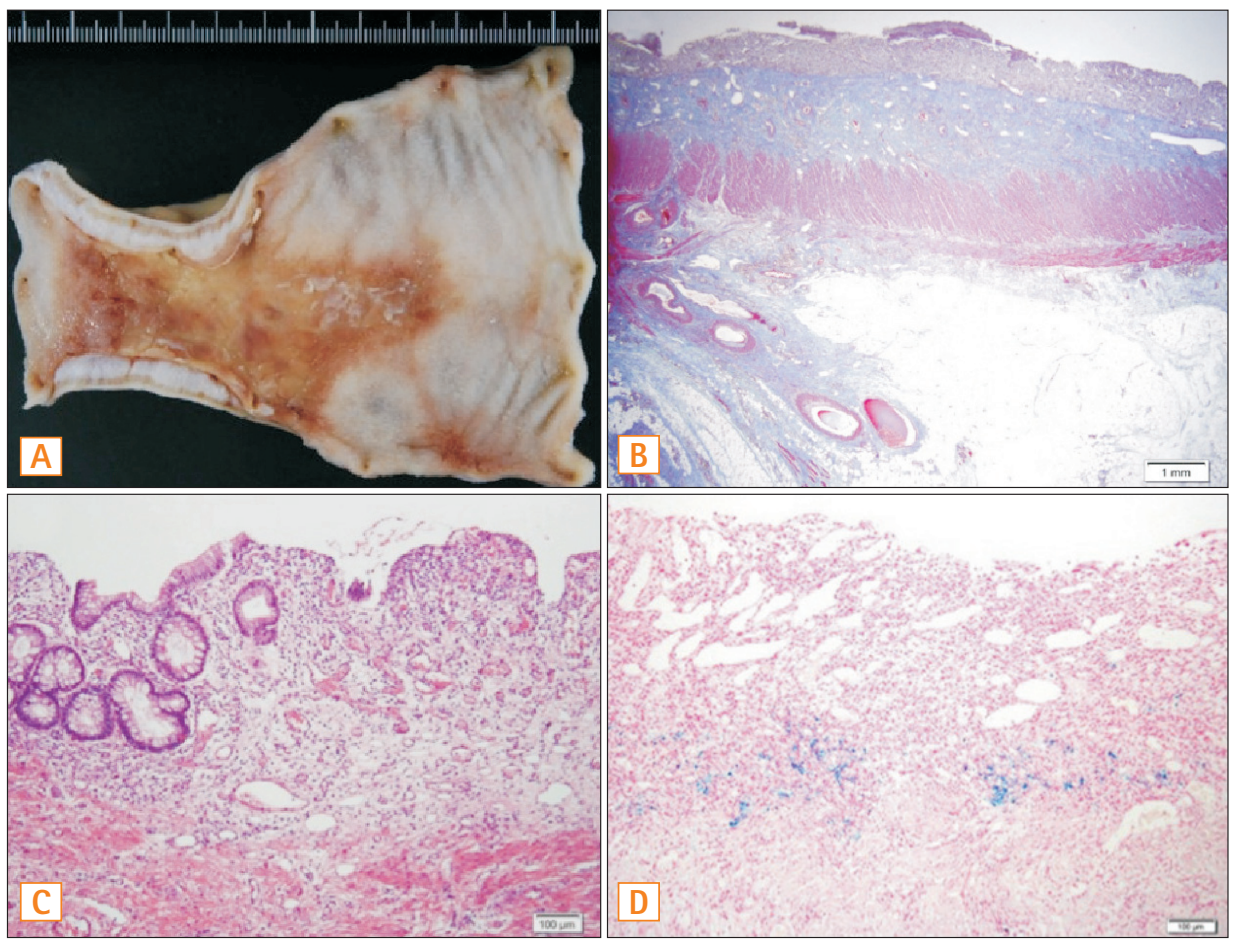

Fig. 3. Histologic findings. (A) Macroscopic findings of the resected specimen revealed a thickened wall and ulcer (anal side is left). (B) Histologic examination revealed transmural ulceration with marked fibrosis, especially in the submucosal layer (Masson's trichrome stain: low-power field). (C) Disappearance of crypt and marked inflammatory cell infiltration in the mucosal layer (HEtE: high-power field). (D) Hemosiderin staining revealed sideroferous cells in the submucosal layers. case reports of IE with intestinal stenosis (date searched: December 2013) (Table 1). In total, we evaluated 33 cases from 26 reports.

Of the 33 cases, 23 patients $(69.7 \%)$ were male and 10 patients $(30.3 \%)$ were female, with a mean age of 61.9 years. As for underlying diseases, 15 cases $(45.4 \%)$ had cardiovascular disease and 7 (21.2\%) had arrhythmia. Four cases (12.1\%) were determined to have no underlying disease. A case series by Sada et al. indicated that $46.4 \%$ of patients with stenotic IE also had underlying disease, including hypertension, ischemic heart disease, arrhythmia, cerebral infarction, or diabetes. ${ }^{4}$ This case series suggests that IE appears to be more common in elderly people with thrombotic conditions, although there were some cases of IE in younger patients without underlying disease. ${ }^{5,6}$

For the clinical presentation of IE, our study revealed that initial symptoms included abdominal pain (93.9\%) or vomiting $(60.6 \%)$, and melena was rare. In addition, 23 patients (69.6\%) had an ileus. The representative clinical course of IE was as follows: sudden onset of symptoms mentioned above, which promptly disappeared under conservative treatment, but recurrence of similar symptoms about 2 months later that required surgical resection of stenosis. ${ }^{4,7}$ The likely reason for this course is that the pathophysiology of IE comprises two phases: (1) an acute phase (transient type) that involves sudden occlusion of the small intestine, and (2) a chronic phase (stenotic type) that involves development of stenosis. The difference may be associated with the degree of ischemia. ${ }^{4}$ Our case already had stenosis and ileus at the point of admission, and thus seemed to be in the chronic phase. Considering the pathophysiology, elucidation of the mechanism of intestinal fibrosis in IE seems crucial for avoiding surgical treatment.

We also evaluated the radiographic findings of IE. Our case series study showed that the characteristic radiographic findings were tubular afferent stenosis with a slightly irregular contour and dilation of the proximal bowel. Thumbprinting in the proximal bowel was observed in our case, which may reflect the concomitant occurrence of various phases of IE. In addition, most cases of IE are located in the ileum.

Enteroscopy also allows for precise examination of the small intestine. Enteroscopy was performed in 11 cases $(33.3 \%)$. Findings associated with IE include circumferential ulcerations and afferent stenosis, which differ from the representative findings of IC (longitudinal ulcers). The region of stenosis may be so narrow that a scope cannot pass through. This difference seems to be attributable to differences in the hemodynamics and tissue reactions to ischemia between the colon and small intestine. The stenosis was located in the jejunum in 10 cases $(30.3 \%)$, and in the ileum in the other 23 cases $(69.7 \%)$. Six cases (18.1\%) had multiple lesions. The mean length of the stenosis was $11.4 \mathrm{~cm}$. Sada et al. reported 


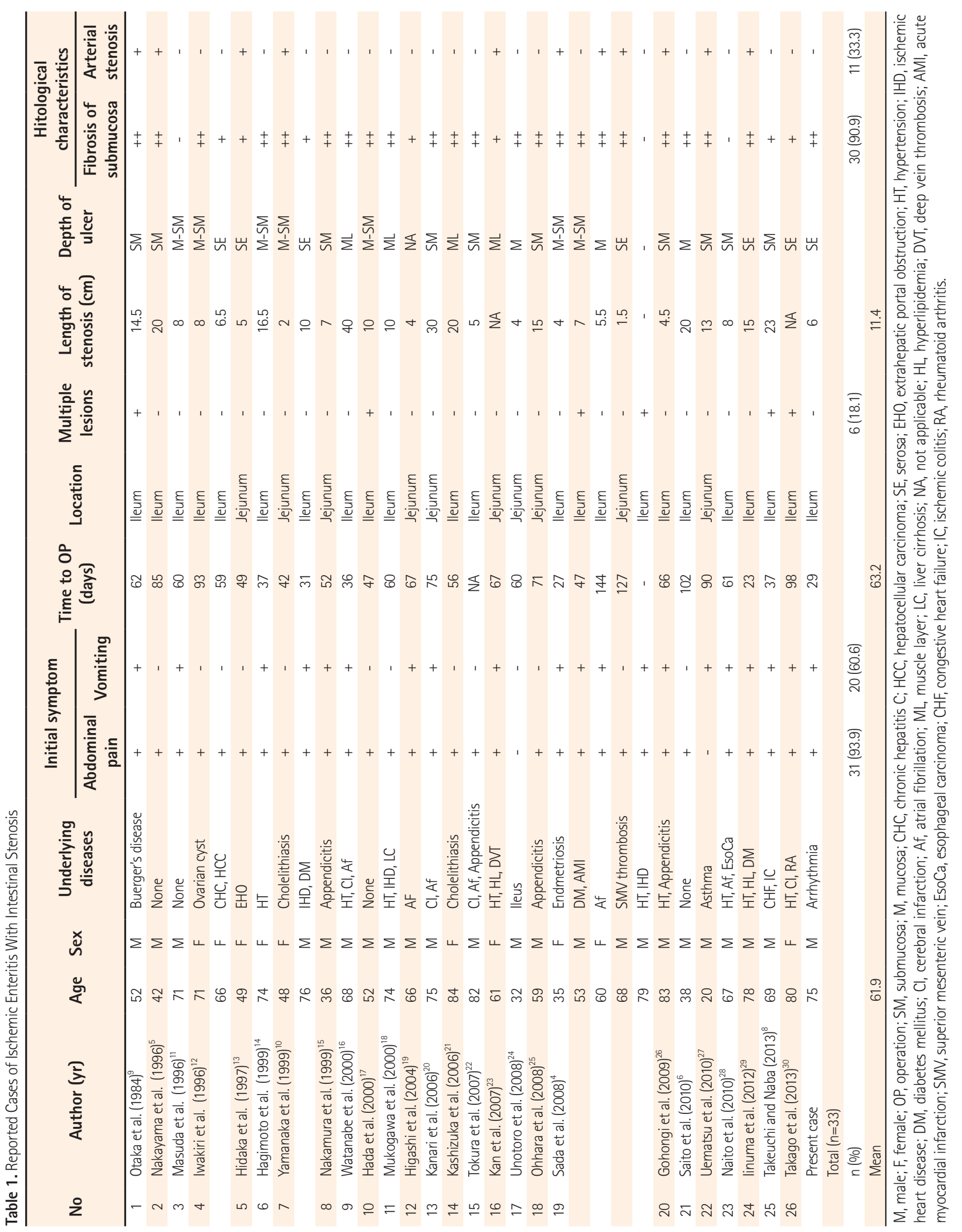


Table 2. Differential Diagnosis Considerations for Stenosis of the Small Intestine

\begin{tabular}{ll}
\hline \multicolumn{1}{c}{ Short segment } & \multicolumn{1}{c}{ Long segment } \\
\hline Tuberculosis & CD \\
NSAIDs-associated enteritis & Ischemic enteritis \\
Adenocarcinoma & Henoch-Schönlein purpura \\
Malignant lymphoma & Intestinal anisakiasis \\
\hline
\end{tabular}

the mean length of the stenosis was $15.3 \mathrm{~cm} .{ }^{4}$ Only one patient who underwent EBD failed to ameliorate symptoms and finally required surgical treatment. ${ }^{8}$ Although EBD may be suitable for cases of IE with short stenosis, there is no evidence regarding the effect of EBD on the intestinal stenosis associated with IE. Surgical resection was required for the stenosis in 32 cases (97.0\%) and the mean time from appearance of initial symptoms to surgery was 63 days.

Histologic examination of the biopsy specimens revealed nonspecific inflammation. Histologic examination of the surgical specimens revealed ulcerations deeper than the submucosa, with marked fibrosis of the submucosal layer and various degrees of inflammatory cell infiltration in most cases, arterial stenosis in 10 cases (30.3\%), and hemosiderinladen macrophages in 11 cases (33.3\%). These histologic characteristics are consistent with chronic ischemic changes. The histologic characteristics of IE as described by Iwashita et al. were as follows: (1) variable depth of ulcers, about half deeper than the submucosa, (2) an ulcer bed consisting of granulated tissue with rich vessels, (3) marked fibrosis of the submucosal layer, (4) severe inflammatory cell infiltration, mainly lymphocytes and plasma cells, (5) various degrees of hemosiderin-laden macrophages, and (6) mild arterial fibromuscular stenosis. ${ }^{2}$

Clinical outcomes were uneventful for most patients and no recurrence of IE was detected. Some patients, however, developed postoperative infection that followed a fatal course: a 52-year-old male patient with Burger's disease died of sepsis and multiple thrombosis 52 days after his operation. ${ }^{9}$ A 76 -year-old male patient who had a history of stroke died of aspiration pneumonia 17 days after his operation. ${ }^{10}$ Considering that most IE patients are relatively older and have underlying disease, IE patients who undergo surgical treatment must be carefully followed up.

For patients with ileal stenotic lesions, such as reported herein, gastroenterologists must consider various diseases (Table 2). The length of intestinal stenoses associated with adenocarcinoma, malignant lymphoma, NSAID-associated enteritis, and tuberculosis is typically short. Adenocarcino-

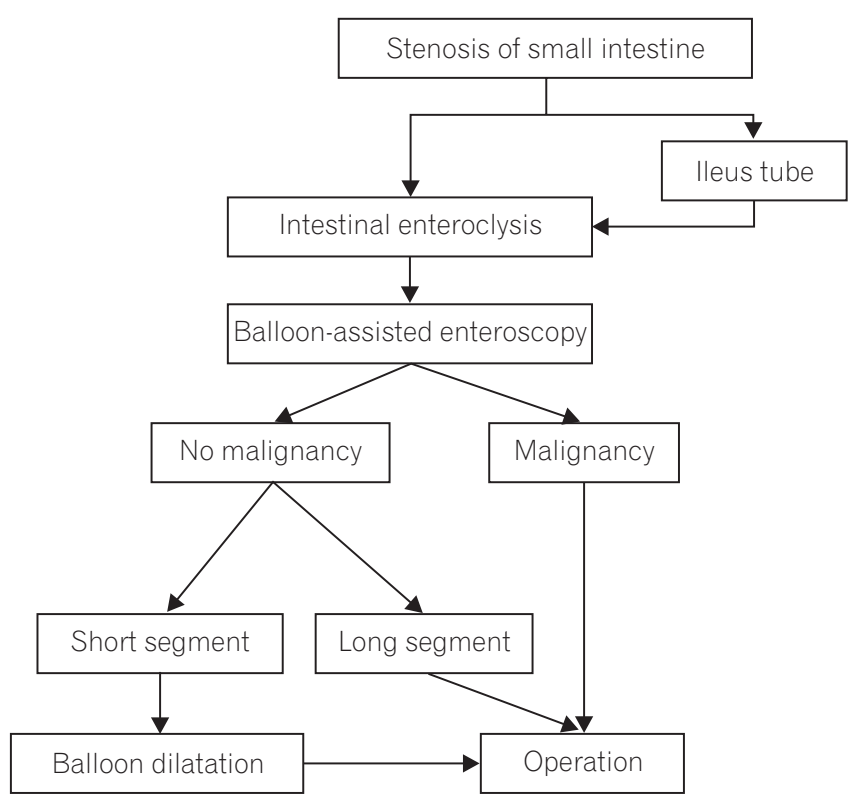

Fig. 4. Proposed management flowchart for stenosis of the small intestine. An ileus tube should be placed in cases of ileus with stenosis of the small intestine. Following one to two weeks of conservative treatment, balloon-assisted enteroscopy should be performed if a definitive diagnosis remains unconfirmed after several examinations, including intestinal enteroclysis. If malignancy is confirmed by histological studies, surgical treatment should be performed. If not, endoscopic balloon dilatation may be considered for a short stenosis. A long stenosis, however, requires surgical treatment.

ma cases often show irregular contours and an overhanging edge, while malignant lymphoma cases show submucosal tumor-like lesions that seldom accompany dilation of the oral intestine. For both diseases, histological examination is necessary to confirm the diagnosis. NSAID-associated enteritis may be accompanied by diaphragm stenosis. Of course, physicians are obligated to evaluate a patient's medication history in this case. The existence of inflammatory polyps or fibrous bands in the surrounding mucosa as well as histologic examination and tissue PCR can facilitate the diagnosis of ileal tuberculosis.

On the other hand, the length of the stenoses observed in cases of CD, IE, Henoch-Schönlein purpura, and intestinal anisakiasis is relatively longer. The diagnosis of HenochSchönlein purpura is simple, because it is accompanied by characteristic purpura in the legs and duodenal lesions such as irregular ulcers, mucosal redness, and petechiae. Thus, esophagogastroduodenoscopy is helpful for diagnosis. The stenoses associated with anisakiasis are much more longer; anisakis larvae can be confirmed by careful enteroclysis. A history of eating raw fish with sudden onset of abdominal 
pain may be suggestive of the disease.

Distinguishing IE from CD is, however, difficult as both diseases are accompanied by tubular stenosis and histologic examination of biopsy specimens may not confirm non-caseous granuloma in all patients with CD. IE occurs in elderly people with several risk factors, and the length of stenosis observed in IE (mean=11.4 $\mathrm{cm}$ ) tends to be longer than that seen in cases of CD. In this regard, the clinical features of IE may be helpful in making a differential diagnosis of patients with ileal stenosis.

In Figure 4, we outline a protocol to manage patients with small bowel stenosis. We strongly recommend the use of enteroscopy to evaluate the stenosis as fully as possible.

In conclusions, IE is uncommon and difficult to diagnose, but increased longevity may lead to more cases of the disease in clinical practice. Therefore, gastroenterologists should keep IE in mind in the differential diagnosis of elderly patients who are determined to have a long ileal stenosis.

\section{REFERENCES}

1. Medina C, Vilaseca J, Videla S, Fabra R, Armengol-Miro JR, Malagelada JR. Outcome of patients with ischemic colitis: review of fifty-three cases. Dis Colon Rectum 2004;47:180-184.

2. Iwashita A, Yao T, Iida M, Yao T, Fuchigami T, Toda T. Clinicopathologic study on ischemic stricture of the small intestine. Stomach and Intestine 1990;25:557-569.

3. Räf LE. Ischaemic stenosis of the small intestine. Acta Chir Scand 1969;135:253-259.

4. Sada M, Kobayashi K, Takeuchi H, et al. Ischemic enteritis. Stomach and Intestine 2008;43:617-623.

5. Nakayama H, Kashiwagi Y, Nishizawa M, Ogawa K, Okui K. A case of ischemic stenosis of small intestine. J Jpn Soc Coloproctol 1997;50:249-253.

6. Saito T, Suzuki T, Watanabe K, et al. A case of ischemic stenosis of the small intestine revealed by double balloon endoscopy. Prog Dig Endosc 2010;77:104-105.

7. Gillet M, Philippe E, Stoebner P, Sava G, Viville C, Grenier JF. Cicatricial stenosis of the small intestine of ischemic origin: 7 cases. Ann Chir 1969;23:481-491.

8. Takeuchi N, Naba K. Small intestinal obstruction resulting from ischemic enteritis: a case report. Clin J Gastroenterol 2013;6:281-286.

9. Ohtaka H, Katsumata T, Takemiya M, et al. A case of ischemic stricture of the small intestine associated with Buerger's disease. Nihon Shokakibyo Gakkai Zasshi 1984;81:2588-2593.
10. Yamanaka H, Matsuda S, Suzuki H. Two resected cases of stenotic type ischemic jejunoileitis. J Jpn Surg Assoc 1999;60:736741.

11. Masuda K, Yamamoto T, Shida H, Ban K, Imanari T, Machida T. Ischmic enteritis causing a small bowel stenosis - Report of a case-. J Jpn Surg Assoc 1996;57:2227-2231.

12. Iwakiri Y, Nakamura K, Sasaki T, et al. Two cases of ischemic enteritis. Jpn J Clin Exp Med 1996;73:1365-1370.

13. Hidaka K, Kobayashi K, Kokubu S. AN operated case of ischemic stricture of the small intestine complicated with extrahepatic portal occlusion. Nihon Shokakibyo Gakkai Zasshi 1997;94:839-844.

14. Hagimoto T, Ohdera K, Nakata K, et al. Serial radiographic change of ischemic enteritis - Report of a case-. J Jpn Soc Coloproctol 1999;52:505-511.

15. Nakamura F, Dohke M, Narita Y, Miyazaki K, Matsunami O, Katoh H. A case of ischemic stricture of the small intestine. J Jpn Surg Assoc 1999;60:129-133.

16. Watanabe T, Hirano M, Kitajima K, Okita K, Yarimizu T, Akizuki $\mathrm{S}$. A case of ischemic enteritis showing a tubular stenosis. Nihon Shokakibyo Gakkai Zasshi 2000;97:191-194.

17. Hada T, Kohno S, Oda Y, Kobayashi I, Ikegami M, Yamazaki Y. Resection and stricturoplasty for ischemic enteritis: a case report. Jpn J Gastroenterol Surg 2000;33:1831-1834.

18. Mukogawa T, Okumura T, Sugimori S, Misaki S, Nakano H. Ischemic stenosis of the small intestine - Report of a case-. J Jpn Surg Assoc 2000;61:675-679.

19. Higashi Y, Nakamura T, Hayashi T, Uno A, Konno H, Nakamura $\mathrm{S}$. A case of ischemic stenosis of the small bowel treated with laparoscopy-assisted surgery. J Jpn Surg Assoc 2004;65:12771280.

20. Kanari M, Fujisawa J, Yukawa N, Nagano A, Matsukawa H, Kawano N. A case of ischemic stenosis of the small intestine. J Jpn Surg Assoc 2006;67:2396-2399.

21. Kashizuka H, Yamamoto M, Nishiwaki H, Hosoi T, Tsutsumi M, Imagawa A. A case of stenotic type ischemic enteritis with hepatic portal venous gas. Jpn J Gastroenterol Surg 2006;39:18501855.

22. Tokura M, Uesaka K, Seima Y, Fujiwara S, Shimada Y. A case of ischemic stricture of the small intestine which could be observed with colonoscopy. J Jpn Surg Assoc 2007;68:2778-2782.

23. Kan H, Furukawa K, Suzuki H, et al. A case of ischemic stenosis of the small intestine revealed by double balloon enteroscopy and resected by laparoscopy-assisted surgery. Jpn J Gastroenterol Surg 2007;40:1514-1519.

24. Unotoro J, Takita N, Suzuki Y, Shiozaki T, Tamazaki S, Tsurumaru M. A case of ischemic stricture of the jejunum due to ischemic enteritis. J Jpn Surg Assoc 2008;69:385-389. 
25. Ohhara M, Miyake H, Kikuchi T, Hara J, Kimizuka K. A case of ischemic stenosis of the small intestine associated with ischemic colitis of the sigmoid colon. J Jpn Surg Assoc 2008;69:19641967.

26. Gohongi T, Ogata T, Nakano Y, Iida H, Gunji N, Orii K. A case of ileus caused by ischemic enteritis of the ileum. J Jpn Surg Assoc 2009;70:2013-2016

27. Uematsu J, Kawai T, Yamamoto K, et al. A case of ischemic enteritis in young age. Prog Dig Endosc 2010;76:86-87.
28. Naito M, Haneda T, Ishiguro T. A case of ischemic enteritis diagnosed by exploratory surgery. Hokkaido J Surg 2010;55:143146.

29. Iinuma N, Yamamoto K, Kubota K, Takagi S. A case of ischemic stenosis of the small intestine. Shinshu Med J 2012;60:365-370.

30. Takago S, Morishita M, Matsunoki A, Arano Y, Iida S, Minato H. A case of stricture type ischemic ileitis associated with ischemic colitis. J Jpn Surg Assoc 2013;74:1538-1542. 